\title{
Interactions between perceptual and numerical space
}

\author{
Peter Kramer • Ivilin Stoianov • Carlo Umiltà • \\ Marco Zorzi
}

Published online: 12 May 2011

(C) Psychonomic Society, Inc. 2011

Interactions between numbers and space have become a major issue in numerical cognition; they suggest that numerical representations might be deeply rooted in cortical networks that also subserve spatial cognition (for reviews, see Hubbard, Piazza, Pinel, \& Dehaene, 2005; Walsh, 2003). The hypothesis that numbers are spatially encoded along a "mental number line" (Moyer \& Landauer, 1967; Restle, 1970) oriented from left to right (for those used to a left-to-right reading direction) is strongly supported by neuropsychological findings. Neglect patients, for example, have a rightward bias when bisecting a physical line in the middle and, remarkably, also a bias toward large numbers (right on the mental number line) when estimating which number is halfway between two others (e.g., responding that 5 is halfway between 2 and 6; Zorzi, Priftis, \& Umiltà, 2002; see also Priftis et al., 2008; Priftis, Zorzi, Meneghello, Marenzi, \& Umiltà, 2006; Rossetti et al., 2004; Umiltà, Priftis, \& Zorzi, 2009, 2010; Vuilleumier, Ortigue, \& Brugger, 2004; Zorzi, Priftis, Meneghello, Marenzi, \& Umiltà, 2006). Healthy subjects show a leftward bias in number line bisection (pseudoneglect; Longo \& Laurenco, 2007), which turns into a rightward bias when "virtual neglect" is induced by repetitive transcranial magnetic stimulation (Göbel, Calabria, Farnè, \& Rossetti, 2006).

P. Kramer $\cdot$ I. Stoianov $\cdot$ C. Umiltà $\cdot$ M. Zorzi $(\triangle)$

Dipartimento di Psicologia Generale, Università di Padova,

Via Venezia 8

35131 Padova, Italy

e-mail: marco.zorzi@unipd.it

C. Umiltà · M. Zorzi

Center for Cognitive Science, University of Padova,

Via Venezia 8,

Padova, Italy
Nicholls and McIllroy (2010), moreover, showed that this leftward bias could be eliminated with right-hand spatial cues, but not with left-hand or neutral ones. Thus, possibly due to their reliance on common parietal circuits subserving space coding and attention (Hubbard et al. 2005), perceptual and numerical spaces appear to be remarkably homeomorphic (Zorzi et al., 2002).

Earlier, reaction time (RT) evidence for interactions between numbers and space was provided by Dehaene, Bossini, and Giraux (1993), who discovered the spatial numerical association of response codes (SNARC) effect, consisting of faster left responses to small numbers and faster right responses to large ones. This phenomenon can be induced with various effectors, such as hands, fingers of the same hand (Priftis et al., 2006), feet (Schwarz \& Müller, 2006), and saccades (Fischer, Warlop, Hill, \& Fias, 2004; Schwarz \& Keus, 2004), and emerges even in parity judgments (odd vs. even) of centrally presented numbers, for which numerical magnitude is task irrelevant (Dehaene et al., 1993). It has been argued (Gevers, Verguts, Reynvoet, Caessens \& Fias 2006; Keus \& Schwarz, 2005) that the SNARC effect arises during selection of a left versus right response. Indeed, the SNARC effect correlates better with response-locked than with stimulus-locked scalp potentials (Gevers, Ratinckx, De Baene \& Fias 2006; Keus, Jenks, \& Schwarz, 2005) and disappears, in parity judgment, when left and right responses are replaced by verbal, nonspatial responses (Keus \& Schwarz, 2005). None of these studies, however, directly support the hypothesis of spatial coding of numbers (see also Proctor \& Cho, 2006; Santens \& Gevers, 2008). Indeed, Gevers et al. (2006b) offered a computational account of the SNARC effect without assuming a spatially organized mental number line: "a specific number is not coded as left or 
right but . . . as either small or large, which in turn activates left or right responses" (p. 41; see also Gevers et al., 2010).

Fischer, Castel, Dodd, and Pratt (2003) found a different type of interaction in a visual cued-detection task in which targets were detected faster if they were presented on the left and preceded by small rather than large numbers, or presented on the right and preceded by large rather than small numbers (see also Casarotti, Michielin, Zorzi, \& Umiltà, 2007; Cattaneo, Silvanto, Battelli, \& PascualLeone, 2009; Galfano, Rusconi, \& Umiltà, 2006; Salillas, El Yagoubi, \& Semenza, 2008). Although response selection was irrelevant, minor modifications of Gevers et al. (2006b) model could account for these results, with an automatic categorization of the central number into small versus large, and a consequent activation of left versus right spatial codes.

Whereas numerical processing may very well affect spatial processing, it is the converse (spatial processing affecting numerical processing) that forces a major revision of Gevers et al. (2006b) model. Stoianov, Kramer, Umiltà, and Zorzi (2008) noted that the spatial coding of visual stimuli is fast and automatic (Lu \& Proctor, 1995; Zorzi \& Umiltà, 1995), whereas the spatial coding of numbers on the mental number line is relatively slow, requiring both perceptual processing (digit recognition) and semantic processing (activation of the mental number line). Stoianov et al. hypothesized that visuospatial priming in nonspatial verbal numerical tasks (which exclude response selection bias) would depend on the concurrent activation of perceptual and numerical spatial representations. Indeed, in both number comparison and parity judgment, Stoianov et al. found that an irrelevant visuospatial cue primes a target number more if the cue follows (backward priming) than if it precedes (forward priming) the target. That is, parity judgments were faster for small than for large numbers when followed by a left-side visual cue and faster for large than for small numbers when followed by a rightside visual cue (an interaction between target magnitude and cue position). Unlike the SNARC effect, this spationumerical interaction between perception and semantics (henceforth, SNIPS) effect occurs before, rather than at, response selection. It thus challenges the ideas of both Gevers et al. and Keus and her colleagues (Keus et al., 2005; Keus \& Schwarz, 2005) and supports the hypothesis that numbers are spatially coded.

Although Stoianov et al. (2008) presented clear evidence that spatial cues can affect numerical targets if the former are presented after the latter, they did not exclude the possibility that this SNIPS effect could peak when cue and target are presented simultaneously. Moreover, in Stoianov et al.'s experiments, only left cues were effective; right cues were not. The authors attributed this odd asymmetry to a concurrent numerical magnitude or size effect (consisting of faster responses to small than to large numbers) that could offset the priming effect. In addition to the magnitude effect, a distance effect was also argued to be in play (consisting of faster responses in number comparison to numbers farther from than to numbers closer to a reference number), and possibly also a parity effect (consisting of different RTs to odd than to even numbers; Nuerk, Wiebke, \& Willmes, 2004).

Here in Experiment 1, using a new method, we show for the first time that the SNIPS effect found by Stoianov et al. (2008) can be experimentally, rather than just statistically, isolated from other numerical effects. This method allows us to test whether left and right spatial cues can both induce the SNIPS effect (as predicted by Stoianov et al., 2008) or whether only left cues are effective (falsifying their account). In Experiment 2, we isolate the peak of the SNIPS effect and test whether it occurs when a spatial cue follows the target (as predicted by Stoianov et al.; for supporting arguments, see Nicholls, Loftus, \& Gevers, 2008) or whether the peak occurs when the two are presented simultaneously (falsifying their account).

\section{Experiment 1}

Here, in conditions with a cue, we expected to obtain both the SNIPS effect and other numerical effects (e.g., magnitude and distance effects). In conditions without a cue, we expected to obtain only the other numerical effects, and not the SNIPS effect. By subtracting the results of the latter conditions from those of the former, we expected to obtain a SNIPS effect untainted by the other numerical effects. Using nonspatial verbal response options, we allow the SNIPS effect to emerge, but not the SNARC effect (which depends on spatially organized response options). By varying the target-to-cue stimulus onset asynchrony (SOA), we also make a first attempt to determine at which SOA the SNIPS effect might peak.

\section{Method}

Subjects A total of 32 naïve, right-handed undergraduates of the Università di Padova (20-28 years of age; 22 women, 10 men) participated for a small fee.

Apparatus An IBM-compatible computer with a 17-in. CRT monitor (75-Hz refresh rate) and MATLAB 7 (Cogent toolbox) were used for the millisecond-precise stimulus presentation. Responses were voice-key recorded with a Sigma Tel soundcard (sensitivity: $-59 \mathrm{dBm}, 80-12000 \mathrm{~Hz}$ ). Voice key onsets were detected, with millisecond precision, with an algorithm similar to Rabiner and Sambur's (1975) 
word boundary detection algorithm. Subsequently, the responses were automatically classified by a pretrained back-propagation neural network as either "Ti," "To," "Te," or "Tu." Exceptional low-confidence classifications of this network were reclassified manually. Missing, multiple, or nonclassifiable responses comprised $1.4 \%$ of the trials.

Design, stimuli, and procedure A completely pseudorandomized within-subjects design was used, comprising three number comparison blocks of 80 trials and three parity judgment blocks of 80 trials. A central dot-shaped fixation point (diameter: $0.2^{\circ}$ ) was visible throughout each trial. A 93-ms central target number (range: $1-9$, excluding 5; Arial font, $1.2^{\circ}$ in height) was presented $506 \mathrm{~ms}$ after trial onset (the fixation dot was superimposed on the target, to aid fixation, but was small enough not to hamper target recognition). In $80 \%$ of the trials, the target was followed by a $93-\mathrm{ms}$ disk-shaped backward cue (diameter: $1.5^{\circ}$ ) that appeared, equally often, $6^{\circ}$ to the left or right of fixation after an SOA of 93, 147, 200, or $253 \mathrm{~ms}$ (i.e., each of these cue conditions, as well as the no-cue condition, all occurred with equal probability). Stoianov et al.'s (2008) goal was to demonstrate the existence of the SNIPS effect. Our present goal, however, was to investigate its characteristics and time course. To facilitate the latter investigation, we - unlike Stoianov et al. and Nicholls and Mclllroy (2010) - avoided temporal overlap between targets and cues. Avoiding this overlap, however, forced us to adopt a shorter target duration than that used by Stoianov et al. All stimuli were white on black, and the viewing distance was $50 \mathrm{~cm}$.

Subjects performed number comparisons between the target and the reference number 5 (smaller vs. larger) and parity judgments (odd vs. even). They were urged to respond as quickly and accurately as possible upon target onset, with either "Ti" to smaller, "To" to larger, "Te" to odd, or "Tu" to even target numbers (with response assignment and task order counterbalanced). These meaningless responses were chosen in order to avoid responses with spatial associations. After a 1,200-ms response deadline, the screen turned blank for a pseudorandom duration between 0.8 and $2 \mathrm{~s}$ (preventing a response rhythm). Half of the subjects were presented with three number comparison followed by three parity judgment blocks, separated by short, self-paced breaks; the other half of the subjects were first presented with the parity judgment blocks, followed by the number comparison ones. A practice block of 24 trials (with feedback) preceded each task.

\section{Results}

We performed repeated measures ANOVAs on the RT data of correct responses after averaging across the counterbalanced response assignments. One subject's responses were excluded because of an unusually high error rate of $10.4 \%$. Of the remaining responses, $0.36 \%$ was excluded as premature (within $200 \mathrm{~ms}$ after target onset) or exceptionally slow (more than 1,100 ms after target onset). Another $0.04 \%$ of the responses were excluded because they were more than three standard deviations slower than the condition average (Ratcliff, 1993), although inclusion did not change the results. After outlier removal, the error rate was $1.8 \%$ and correlated positively with RT $(r=.22, p<.01)$.

We first performed an ANOVA on all of the cuepresent data (Table 1) and found a task effect: Responses were slower in parity judgments $(594 \mathrm{~ms})$ than in number comparisons $(572 \mathrm{~ms})\left[F(1,30)=6.4, p=.02, \eta_{\mathrm{p}}{ }^{2}=.18\right]$. We also found a target magnitude effect: Responses were slower for targets larger than $5(588 \mathrm{~ms})$ than for targets
Table 1 Mean reaction times and SNIPS effects (in milliseconds) in Experiments 1 and 2

\begin{tabular}{|c|c|c|c|c|c|c|c|}
\hline \multirow[b]{2}{*}{ Experiment } & \multirow[b]{2}{*}{ SOA } & \multicolumn{2}{|c|}{ Small Magnitude } & \multicolumn{2}{|c|}{ Large Magnitude } & \multicolumn{2}{|c|}{ SNIPS } \\
\hline & & Left Cue & Right Cue & Left Cue & Right Cue & M & SE \\
\hline \multirow[t]{4}{*}{ 1. Parity judgment } & 93 & 576 & 584 & 609 & 596 & 21 & 9 \\
\hline & 147 & 592 & 597 & 604 & 594 & 16 & 10 \\
\hline & 200 & 598 & 588 & 600 & 593 & -4 & 13 \\
\hline & 253 & 586 & 586 & 594 & 602 & -7 & 9 \\
\hline \multirow[t]{4}{*}{ 1. Number comparison } & 93 & 555 & 574 & 586 & 565 & 40 & 14 \\
\hline & 147 & 566 & 574 & 584 & 578 & 15 & 11 \\
\hline & 200 & 566 & 571 & 578 & 568 & 15 & 7 \\
\hline & 253 & 560 & 576 & 577 & 578 & 14 & 13 \\
\hline \multirow[t]{4}{*}{ 2. Parity judgment } & 0 & 568 & 560 & 574 & 581 & -15 & 10 \\
\hline & 40 & 575 & 570 & 574 & 571 & -2 & 8 \\
\hline & 80 & 572 & 585 & 579 & 574 & 18 & 12 \\
\hline & 120 & 569 & 588 & 576 & 570 & 25 & 8 \\
\hline
\end{tabular}


smaller than $5(578 \mathrm{~ms})\left[F(1,30)=15.7, p<.001, \eta_{\mathrm{p}}{ }^{2}=.34\right]$. This target magnitude effect did not interact with task $[F(1$, $30)=0.1, p=.76]$ but did interact with cue position [left vs. right; $\left.F(1,30)=10.9, p<.01, \eta_{\mathrm{p}}{ }^{2}=.27\right]$ : the SNIPS effect (for details, see the next two paragraphs). The SNIPS effect did not interact with task, but did interact with target-to-cue SOA $\left[F(3,90)=3.0, p=.05, \eta_{\mathrm{p}}{ }^{2}=.09\right]$ and only appeared at the two shortest SOAs [respectively, $F(1,30)=14.3$, $p=.001, \eta_{\mathrm{p}}{ }^{2}=.32$, and $\left.F(1,30)=4.9, p=.04, \eta_{\mathrm{p}}{ }^{2}=.14\right]$.

Because the SNIPS effect did not interact with task (number comparison vs. parity judgment), we averaged across this variable. This allowed us to use the resulting gain in statistical power and split the target magnitude categories from two (numbers smaller vs. larger than 5) into four (1 and 2, 3 and 4, 6 and 7, 8 and 9). Next, to isolate the SNIPS effect from other numerical effects, we subtracted the results obtained in conditions with and without a cue from each other, and performed another ANOVA. Because there is no target-to-cue SOA in the no-cue condition, SOA could not be retained as a variable. For the conditions with a cue, we averaged across the two SOAs that produced the SNIPS effect; the other two SOAs were no longer considered. Once again, we found a spationumerical interaction between target magnitude and cue position [the SNIPS effect; see Fig. $1 ; F(3,90)=7.2, p<.001, \eta_{\mathrm{p}}{ }^{2}=.19$ ]. This time, though, unlike in Stoianov et al. (2008), the SNIPS effect was untainted by any other numerical effect. (There were no significant, or marginal, main effects; both $F_{\mathrm{S}}<0.5$.)

We defined as compatible left cuing of targets smaller than 5 and right cuing of targets larger than 5; all other cuing was defined as incompatible. Planned comparisons revealed that, overall, responses were $8 \mathrm{~ms}$ slower after incompatible cuing than after no cuing $[F(1,30)=5.2, p=.03$, two-tailed,

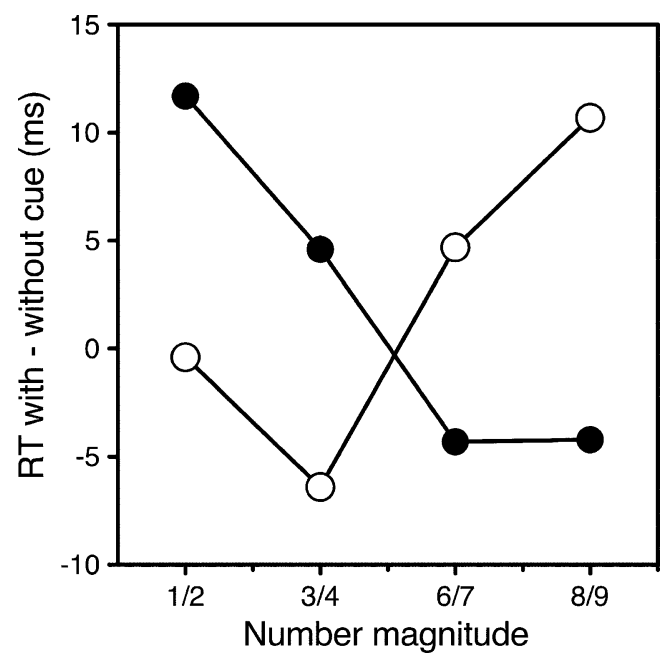

Fig. 1 Mean difference scores for reaction times (RTs) obtained with minus without either a left cue (open symbols) or a right cue (closed symbols) $\left.\eta_{\mathrm{p}}{ }^{2}=.15\right]$, but not significantly faster after compatible cuing than after no cuing $[4 \mathrm{~ms}$ faster, but $F(1,30)=1.2, p=.28$, two-tailed]. For each of the magnitudes separately, responses to small magnitudes $(1 / 2$ and $3 / 4)$ were slower when right than when left cued (respectively, 12 and $11 \mathrm{~ms}, p \mathrm{~s}<.05$, onetailed, both $\left.\eta_{\mathrm{p}}{ }^{2} \mathrm{~s}=.12\right)$. Conversely, responses to large magnitudes (6/7 and 8/9) were slower when left than when right cued (respectively, 9 and $15 \mathrm{~ms}, p \mathrm{~s}<.05$, one-tailed, $\eta_{\mathrm{p}}{ }^{2} \mathrm{~s}=.09$ and .16). Importantly, after the subtraction procedure, it turned out that left and right cues were both (about equally) effective in producing the SNIPS effect (see Fig. 1). (The means for the four magnitude categories $[1 / 2,3 / 4,6 / 7$, and $8 / 9]$ were $569,581,590$, and $587 \mathrm{~ms}$ for the no-cue condition; $569,575,594$, and $598 \mathrm{~ms}$ for the left-cue condition; and 581, 586, 585, and $583 \mathrm{~ms}$ for the right-cue condition.)

In principle, the mere presence of a cue could either alert or distract subjects. The presence of a cue by itself, howeverunlike its presence in a specific position - cannot produce any of the effects reported here. Moreover, the difference between our conditions with a cue $(584 \mathrm{~ms}$, averaged across left and right cues) and without a cue $(582 \mathrm{~ms})$ was minute and far from significant $[t(30)=0.6, p=.52$, two-tailed, considering only the two effective-i.e., the shortest-SOAs].

\section{Experiment 2}

Stoianov et al.'s (2008) explanation of the SNIPS effect requires that it peak at a positive target-to-cue SOA. Consistent with their hypothesis, they found only backward, and no forward, priming effects. However, they did not exclude the possibility that the SNIPS effect might peak at a $0-\mathrm{ms}$ SOA, and if found, this would falsify Stoianov et al.'s contention. Experiment 1 successfully isolated the SNIPS effect from other numerical effects but did not succeed in isolating the peak of its time course. That is, the SNIPS effect was observed at SOAs of 93 and $147 \mathrm{~ms}$, and not at SOAs of 200 and $253 \mathrm{~ms}$, but these results do not exclude the possibility that the peak of the SNIPS effect could be located at an SOA of $0 \mathrm{~ms}$. The present Experiment 2, with somewhat different stimuli and a different range of SOAs (including one of $0 \mathrm{~ms}$ ), represented a second attempt at finding this peak.

Method

Subjects A total of 16 naïve, right-handed undergraduates of the Università di Padova (20-34 years of age; 11 women, 5 men) participated for a small fee.

Apparatus, stimuli, and procedure The same apparatus and software were used as in Experiment 1. The stimuli were 
also similar, but were presented on a gray background (1) to minimize possible effects of the screen's phosphor persistence and (2) to drown out any visible persistence trail within the subjects' visual system (both of which could potentially affect the SNIPS effect's time course). A 40-ms target (height: $1.0^{\circ}$ ) was presented and an $80-\mathrm{ms}$ cue (diameter: $\left.1.5^{\circ}\right) 5^{\circ}$ away from fixation. The target-to-cue SOAs were $0,40,80$, and $120 \mathrm{~ms}$, so that the target and cue overlapped in time only for the first SOA and not for the last three. Since both the 40-ms and 80-ms target-to-cue SOAs were shorter than the SOAs in Experiment 1, avoiding temporal overlap forced us to choose a shorter target duration here than in Experiment 1. Viewing distance was $75 \mathrm{~cm}$. The task was a parity judgment with the nonspatial vocal responses of "Te" for even and "Tu" for odd, or the converse (counterbalanced across subjects). The experiment consisted of a practice block of 24 trials and six blocks of 64 trials. A completely pseudorandomized within-subjects design was used. Missing, multiple, or nonclassifiable responses comprised $2.8 \%$ of the trials.

\section{Results}

The responses of 1 male subject were excluded because of an unusually high error rate of $8 \%$. The error rate in the remaining data was $1.6 \%$ and correlated positively with RT $(r=.17, p<.01)$. No responses were faster than $200 \mathrm{~ms}$ or slower than $1,100 \mathrm{~ms}$, but $0.7 \%$ of them were excluded because they were more than three standard deviations slower than the condition average (inclusion of the excluded data did not change the results).

A repeated measures ANOVA revealed a spationumerical interaction between cue position, target magnitude, and target-to-cue SOA $\left[F(3,42)=3.5, p=.03, \eta_{\mathrm{p}}{ }^{2}=.19\right]$. No other effects were significant (or marginally significant). Planned contrasts revealed a significant effect at the longest SOA (120 ms), but no (marginally) significant effects at the shorter SOAs. At the SOA of $120 \mathrm{~ms}$, responses were faster to small left-cued than to small right-cued numbers, and slower to large left-cued than to large right-cued numbers [the SNIPS effect; see Table $1 ; F(1,14)=9.5$, $\left.p<.01, \eta_{\mathrm{p}}{ }^{2}=.41\right]$. Thus, the SNIPS effect peaked at a positive, and not a zero, target-to-cue SOA.

\section{Discussion}

With their SNIPS effect, Stoianov et al., (2008) supported the existence of a spatially organized mental number linethe only RT evidence to date that cannot be discounted by alternative models. Earlier, a SNARC effect had been found (Dehaene et al., 1993), but it has been related to response selection and does not require a spatial representation of numbers (Gevers et al., 2010; Gevers et al. 2006b). What renders the SNIPS effect controversial, and different from the SNARC effect, is that it emerges even when response selection effects are excluded (by the use of nonspatial verbal response options).

The SNIPS effect emerges when relatively slowly processed numerical targets (which require both perceptual and semantic processing) and relatively quickly processed spatial cues (which require only perceptual processing) activate their respective spatial representations concurrently. In Experiment 1, we first replicated this controversial effect, and then introduced a method to isolate it from other numerical effects. To this end, we subtracted the results obtained without spatial cues from those obtained with them. The SNIPS effect was still observed, and as required by Stoianov et al.'s (2008) account of it, we found that left and right spatial cues are both (about equally) effective in producing the SNIPS effect. Left cues delayed responses to large numbers, and right cues delayed responses to small numbers. There was no significant response facilitation in any condition. The mere presence of a cue by itself had no effect. Hence, we concluded that the SNIPS effect is predominantly inhibitory.

In Experiment 2, we isolated the peak of the SNIPS effect and, as required by Stoianov et al.'s (2008) account, we found that it occurs when a cue follows a target, not when the two are presented concurrently. In fact, we obtained the SNIPS effect only at a target-to-cue SOA of $120 \mathrm{~ms}$. Quickly processed spatial cues affect more slowly processed numerical targets if they are presented with a sufficiently large target-to-cue SOA. The faster the cue is processed, the larger should be the required SOA. If our most briefly presented cues triggered the visual system's fastest transient channels, then perhaps this might explain why the SNIPS effect required the largest SOA in Experiment 2 (cue duration $=80 \mathrm{~ms}$ ), a smaller SOA in Experiment 1 (cue duration $=93 \mathrm{~ms}$ ), and the smallest $\mathrm{SOA}$ in Stoianov et al. (cue duration $=118 \mathrm{~ms}$ ). Since the experiments differed in more than just cue duration, however, we must leave this issue for future study.

Taken together, our results corroborate Stoianov et al.'s (2008) claim that spationumerical interactions can occur before response selection, and corroborate their account of these interactions. The results thus reconcile neuropsychological and RT studies and support the hypothesis that numbers are represented along a left-to-right spatially organized mental number line (see, e.g., Zorzi et al., 2002). We have shown that if the representations of perceptual space and numerical space are concurrently activated, a SNIPS effect emerges, and we have shown that this effect is equally well induced by both left and right irrelevant spatial 
cues, and that it peaks when a cue follows a target and not when the cue is presented concurrently with the target.

Although the precise mechanism underlying the SNIPS effect remains to be investigated, the hypothesis that the effect reflects functional-anatomical overlap between the domains of space and number (Walsh, 2003) seems at odds with the finding that the effect is largely inhibitory. In contrast, the effect induced by the spatial cues fits well with the evidence from neglect patients that number processing is strongly biased by the allocation of spatial attention (Umiltà et al., 2009, for review). Accordingly, Hubbard et al. (2005) and Loetscher, Bockisch, and Brugger (2008) suggested that spationumerical interactions arise from common parietal circuits for attention to external physical, and internal numerical, spaces.

Author Note This study was supported by the European Research Council (Grant 210922 to M.Z.) and by the Italian Ministry of Education, University and Research (PRIN 2007 to C.U. and PRIN 2008 to M.Z.). Thanks to Alessandra Della Valle for data collection.

\section{References}

Casarotti, M., Michielin, M., Zorzi, M., \& Umiltà, C. (2007). Temporal order judgment reveals how number magnitude affects visuospatial attention. Cognition, 102, 101-117.

Cattaneo, Z., Silvanto, J., Battelli, L., \& Pascual-Leone, A. (2009). The mental number line modulates visual cortical excitability. Neuroscience Letters, 462, 253-256.

Dehaene, S., Bossini, S., \& Giraux, P. (1993). The mental representation of parity and numerical magnitude. Journal of Experimental Psychology: General, 122, 371-396.

Fischer, M. H., Castel, A. D., Dodd, M. D., \& Pratt, J. (2003). Perceiving numbers causes spatial shifts of attention. Nature Neuroscience, 6, 555-556.

Fischer, M. H., Warlop, N., Hill, R. L., \& Fias, W. (2004). Oculomotor bias induced by number perception. Experimental Psychology, 51, 91-97.

Galfano, G., Rusconi, E., \& Umiltà, C. (2006). Number magnitude orients attention, but not against one's will. Psychonomic Bulletin \& Review, 13, 869-874.

Gevers, W., Ratinckx, E., De Baene, W., \& Fias, W. (2006). Further evidence that the SNARC effect is processed along a dual-route architecture: Evidence from the lateralized readiness potential. Experimental Psychology, 53, 58-68. doi:10.1027/16183169.53.1.58

Gevers, W., Santens, S., Dhooge, E., Chen, Q., van den Bossche, L., Fias, W., et al. (2010). Verbal-spatial and visuospatial coding of number-space interactions. Journal of Experimental Psychology: General, 139, 180-190.

Gevers, W., Verguts, T., Reynvoet, B., Caessens, B., \& Fias, W. (2006b). Numbers and space: A computational model of the SNARC effect. Journal of Experimental Psychology: Human Perception and Performance, 32, 32-44.

Göbel, S.M., Calabria, M., Farnè, A., \& Rossetti, Y. (2006). Parietal rTMS distorts the mental number line: Simulating "spatial" neglect in healthy subjects. Neuropsychologia, 44, 860-868. doi:10.1016/j.neuropsychologia.2005.09.007
Hubbard, E. M., Piazza, M., Pinel, P., \& Dehaene, S. (2005). Interactions between numbers and space in parietal cortex. Nature Reviews Neuroscience, 6, 435-448.

Keus, I. M., Jenks, K. M., \& Schwarz, W. (2005). Psychophysiological evidence that the SNARC effect has its functional locus in a response selection stage. Cognitive Brain Research, $24,48-56$

Keus, I. M., \& Schwarz, W. (2005). Searching for the functional locus of the SNARC effect: Evidence for a response-related origin. Memory \& Cognition, 33, 681-695.

Loetscher, T., Bockisch, C. J., \& Brugger, P. (2008). Looking for the answer: The mind's eye in number space. Neuroscience, 151, $725-729$.

Longo, M. R., \& Laurenco, S. F. (2007). Spatial attention and the mental number line: Evidence for characteristic biases and compression. Neuropsychologia, 45, 1400-1407.

Lu, C. H., \& Proctor, R. W. (1995). The influence of irrelevant location information on performance: A review of the Simon and spatial Stroop effects. Psychonomic Bulletin \& Review, 2, 174 207.

Moyer, R., \& Landauer, T. (1967). Time required for judgments of numerical inequality. Nature, 215, 1519-1520.

Nicholls, M. E. R., Loftus, A. M., \& Gevers, W. (2008). Look, no hands: A perceptual task shows that number magnitude induces shifts of attention. Psychonomic Bulletin \& Review, 15, 413418.

Nicholls, M. E. R., \& McIllroy, A. M. (2010). Spatial cues affect mental number line bisections. Experimental Psychology, 57, 315-319.

Nuerk, H. C., Wiebke, I., \& Willmes, K. (2004). Notational modulation of the SNARC and the MARC (linguistic markedness of response codes) effect. Quarterly Journal of Experimental Psychology, 57A, 835-863.

Priftis, K., Piccione, F., Giorgi, F., Meneghello, F., Umiltà, C., \& Zorzi, M. (2008). Lost in number space after right brain damage: A neural signature of representational neglect. Cortex, 44, 449453.

Priftis, K., Zorzi, M., Meneghello, F., Marenzi, R., \& Umiltà, C. (2006). Explicit versus implicit processing of representational space in neglect: Dissociations in accessing the mental number line. Journal of Cognitive Neuroscience, 18, 680-688.

Proctor, R. W., \& Cho, Y. S. (2006). Polarity correspondence: A general principle for performance of speeded binary classification tasks. Psychological Bulletin, 132, 416-442.

Rabiner, L. R., \& Sambur, M. R. (1975). An algorithm for determining the endpoints of isolated utterances. Bell System Technical Journal, 54, 297-315.

Ratcliff, R. (1993). Methods for dealing with reaction time outliers. Psychological Bulletin, 114, 510-532.

Restle, F. (1970). Speed of adding and comparing numbers. Journal of Experimental Psychology, 83, 274-278. doi:10.1037/h0028573

Rossetti, Y., Jacquin-Courtois, S., Rode, G., Ota, H., Michel, C., \& Boisson, D. (2004). Visuo-manual adaptation improves number bisection in unilateral neglect. Psychological Science, 15, 426430.

Salillas, E., El Yagoubi, R., \& Semenza, C. (2008). Sensory and cognitive processes of shifts of spatial attention induced by numbers: An ERP study. Cortex, 44, 406-413.

Santens, S., \& Gevers, W. (2008). The SNARC effect does not imply a mental number line. Cognition, 108, 263-270.

Schwarz, W., \& Keus, I. M. (2004). Moving along the mental number line: Comparing SNARC effects with saccadic and manual response. Perception \& Psychophysics, 66, 651-664.

Schwarz, W., \& Müller, D. (2006). Spatial associations in number related tasks: A comparison of manual and pedal responses. Experimental Psychology, 53, 4-15. 
Stoianov, I., Kramer, P., Umiltà, C., \& Zorzi, M. (2008). Visuospatial priming of the mental number line. Cognition, 106, 770-779.

Umiltà, C., Priftis, K., \& Zorzi, M. (2009). The spatial representation of numbers: Evidence from neglect and pseudoneglect. Experimental Brain Research, 192, 561-569.

Umiltà, C., Priftis, K., \& Zorzi, M. (2010). Visuo-spatial representation of number magnitude. In V. Coltheart (Ed.), Tutorials in visual cognition (pp. 337-348). New York: Psychology Press.

Vuilleumier, P., Ortigue, S., \& Brugger, P. (2004). The number space and neglect. Cortex, 40, 399-410.
Walsh, V. (2003). A theory of magnitude: Common cortical metrics of time, space and quantity. Trends in Cognitive Sciences, 7, 483488.

Zorzi, M., Priftis, K., Meneghello, F., Marenzi, R., \& Umiltà, C. (2006). The spatial representation of numerical and nonnumerical sequences: Evidence from neglect. Neuropsychologia, 44, 1061-1067. doi:10.1016/j.neuropsychologia.2005.10.025

Zorzi, M., Priftis, K., \& Umiltà, C. (2002). Neglect disrupts the mental number line. Nature, 417, 138-139.

Zorzi, M., \& Umiltà, C. (1995). A computational model of the Simon effect. Psychological Research, 58, 193-205. 